\title{
Performance Evaluation of AODV, DSR and OLSR in MANET using Opnet Simulator
}

\author{
Zahraa E. Mohamed \\ Faculty of Science, \\ Mathematics Department, \\ Zagazig University, \\ Zagazig, Egypt
}

\author{
Mahmoud Atef \\ High Institute for \\ Engineering and Technology, \\ Al-Obour, Cairo, Egypt
}

\begin{abstract}
Mobile Ad hoc Networks (MANETs) is a combination of wireless mobile nodes that exchanges information and dynamically form a network of self-configuring and selforganizing without any fixed infrastructure or centralized administration. MANETs is very important in the researches of the military and civilian applications. Routing is a major part in the success of any communication between these mobile nodes, therefor a routing protocols play an important role in finding an efficient and reliable route between mobile nodes from source to destination. This paper focuses on the performance analysis of these well-known routing protocols named DSR, AODV and OLSR and it evaluates the performance based on the rate of file transfer protocol (FTP) with (medium load) traffic and varying the number of nodes in a two different scenarios to assess the performance of each protocol and determine the best. In this work, the performance comparison between the AODV, DSR and OLSR routing protocol in terms of data dropped, end to end delay, throughput and routing overhead have been performed by using OPNET version 17.5 simulator. The simulation results showed that OLSR protocol is mainly more suitable for large dense networks.
\end{abstract}

\section{General terms}

Mobile ad hoc network, routing protocols, Opnet simulator

\section{Keywords}

MANETs, AODV, DSR, OLSR, FTP, OPNET.

\section{INTRODUCTION}

A multi-hop network is a type of wireless network that uses more than one wireless node to transmit its information from a source node to a destination node. These nodes freely and dynamically self-organize themselves. Mobile ad-hoc network (MANET) is a type of multi-hop network where each node in this network, is free to move randomly and independently in any direction and hence, frequently the links between the nodes change with unpredictable manner [1]. Each node dynamically discovers other nodes such that, they can communicate together directly. A mobile ad hoc network (MANET) is a self-configuring and selforganizing without any fixed infrastructure or centralized administration. A mobile node not only transmits its own packets, but also forwards packets for other mobile nodes, this mean each one of which is equipped with a radio transmitter and a receiver [2][3].

There are various ways to communicate remotely like Zigbee, Wimax, WiFi and Bluetooth and etc. All the previously mentioned systems give a communications short range. There are many areas that need long range wireless communication such as earthquake relief, military field and other areas of the emergency communications and here MANET plays an important role [4]. Therefore, in recent years MANETs have become an important subject of active research. An ad hoc routing protocol is a convention or standard which organizes the relations between nodes and controls that decide how nodes choose the path to send data packet between the devices with each other in a MANET system [5][ 6][ 7]. There are a several protocols for MANETs which are divided into three categories, namely reactive routing protocol, proactive routing protocol and hybrid routing protocol depending to their performance and functionality. Reactive routing protocols are also known on demand routing protocol for example AODV, DSR, LAR, TORA and etc. proactive routing protocols are also known table driven routing protocols for example FSR, DSDV, OLSR, WRP and etc.

In recent years, most the researches focus on the performance comparisons of routing protocols in MANETs using Ns-2 and OMNeT++ network simulators. There are many works has been done on the performance comparison area between most of the existing routing protocols for MANETs. A review of the performance of the routing protocols was performed by

P. Kuppusamy et al. [8]. They attempted to evaluate the performance study for several MANET protocols and study the performance assess of a three ad hoc routing protocols (OLSR, AODV and TORA) by increasing the number of nodes in the network. The routing overhead, PDR and end-toend delay are a three performance metrics that have been used for study the performance of the mentioned protocols. The results showed that in dense networks the routing protocol AODV and TORA have a good performance compared with OLSR protocol in terms of packet delivery ratio. R. Kaur et al. [9] studied the performance of three routing protocols AODV, DSDV and OLSR. The Packet Delivery Ratio, throughput and End-to-End Delay are the performance metrics that have been used to analysis the performance between above protocols. OLSR perform better than DSDV and AODV in a three performance metric that used in this study. The performance evaluation of three routing protocols OLSR, GRP and TORA under different nodes number has been done by $\mathrm{H}$. kaur et al. [10]. The performance metrics that have been used in this work are load, delay, routing overhead and throughput. They have concluded that GRP performs better than OLSR and TORA in terms of routing overhead and delay whereas OLSR performs best in terms of throughput and load. N. P. Bobade1 et al. [11] presented the performance of AODV and DSR is evaluated with respect to performance metrics like throughput, PDF, end-to-end delay and NRL by varying network size. The authors have been concluded that AODV is the best performance in terms of throughput and PDF whereas DSR performs best in terms of end-to-end delay 
with varying network size. Comparison between two reactive protocols which are AODV and DSR and two proactive which are OLSR and DSDV have been presented by A. Al-Baseer et al. [12]. In this work the performance of four well-known routing protocols was evaluated based on ad hoc network size (number of nodes). They have used three metrics to evaluate the performance between above protocols which are the packet delivery ratio (PDR), the collision and end-to-end delay with different scenarios. The simulation results under five proposed scenarios ( $8,16,32,40$ and 80 nodes) had been shown that AODV and OLSR accomplished a good performance compared with DSDV and DSR in all given metrics.

Having done so much study on the previous related work, it was discovered that much have not been done in evaluating the performance of AODV, DSR and OLSR together. Research effort have not focused much in evaluating their performance under a variable number of nodes. Moreover, most of the works described above used $\mathrm{n} 2$ as a simulator whereas this work will be done in OPNET version 17.5.

The primary objective of this paper is to investigate the characteristics of mobile ad-hoc network protocols, and also evaluate and compare the performance of AODV, DSR, and OLSR protocols for wireless ad-hoc network. The first two protocols have been selected from Reactive category, namely AODV and DSR and the third protocol has been selected from a proactive category namely OLSR. The process of evaluation in this paper is based on the rate of file transfer protocol (FTP) with (medium load) traffic and varying the number of nodes in different two scenarios to assess the performance of each protocol. The rest of the paper is organized as follows: Section 2 describes a brief idea on the different routing protocols used for performance analysis in this paper. Section 3 explains the simulation parameters, simulation setup and describes the performance metrics, data dropped, delay, throughput and routing overhead of considered protocols. The simulation results and analysis is described in section 4 and in section 5 concluded our work.

\section{ROUTING PROTOCOL IN MANET}

\subsection{Ad Hoc On-Demand Distance Vector Routing (AODV)}

AODV is a combination of on-demand and distance vector. AODV minimizes the number of required broadcasts by creating routes in an on-demand manner. When a source node desires to send data to other destination node, it needs to initiate a path discovery process to locate the other node. AODV performs two operations Route Discovery and Route Maintenance. Route discovery is done in AODV by broadcasting the Route Request (RREQ) message from the source node to neighbor nodes, which then forward the request to their neighbors, and so on, until the destination is located. When the RREQ reaches a node with route to destination, the destination node is responds and creates again a Route Replay (RREP) back to the source node and contains the number of hops that are require to reach the destination. AODV maintains all the route information in the form of routing table and also uses the destination sequence number to keep its information updated and to try to be loop free and avoidance any broken routes [13].

\subsection{Dynamic Source Routing (DSR)}

DSR is a routing protocol that is established on the concept of source routing an also named on-demand routing protocol. Each node in network maintains a route cache with route entries which are continuously updated. Each node maintains a route caches containing the source routes that it is aware of. The node updates entries in the route cache continuously. The advantage of DSR is that no periodic routing packets are required. It is used to updates its route caches by finding new routes [14]. DSR has two major phases: route discovery and route maintenance.

\subsection{Optimized Link State Routing (OLSR)}

OLSR is the most suitable example of a table driven proactive routing protocol for mobile ad hoc networks. It is an optimization version of a pure link state uses the concept of Multipoint Relay to generating and forwarding topology information and reduces the generated overhead to all nodes in to the entire network by limiting the number of broadcasts [15]. OLSR protocol uses four types of messages they are HELLO packets message, Topology Control (TC), Multiple Interface Declaration messages and Host and Network Association message to discover the neighbour's and gaining information about them. MPR nodes it is the only responsible of route broadcast packet in the network in order to reduce the size of broadcast. OLSR is more suitable for large and dense networks than AODV and DSR.

\section{SIMULATION ENVIRONMENT AND SETUP}

In order to carry out the performance comparison between the three routing protocols that have been described in section 2, OPNET (Optimized Network Engineering Tool) Modeler version 17.5 was used for this purpose. Simulation experiments were performed under two main scenarios that have been created and analyzed by varying the number of nodes, one has a few density of nodes and other has a high density of nodes. Table 1 shows the simulation parameters that used to evaluation the performance of routing protocols in this paper.

Table 1: Simulation parameters for MANETs

\begin{tabular}{|c|c|}
\hline Parameter Name & Parameter Value \\
\hline Routing Protocol & AODV ,DSR, OLSR \\
\hline Environment size & $1000 \mathrm{~m}$ x $1000 \mathrm{~m}$ \\
\hline Simulation Time & 15 Minutes $(900 \mathrm{sec})$ \\
\hline Pause time & 0 second \\
\hline Data packet size (bits) & $512(64$ Bytes $)$ \\
\hline Speed of node & $5 \mathrm{~m} / \mathrm{sec}$ \\
\hline Packets Rate & 4 packets/s \\
\hline Packets type & CBR \\
\hline Traffic Type & FTP(medium load) \\
\hline Simulator & OPNET 17.5 \\
\hline Number Of Nodes & $25,35,85,100$ \\
\hline
\end{tabular}

\subsection{Simulation Setup}

For an optimized working of the network, one WLAN server was configured with medium FTP traffic for both scenarios where the size of the FTP file was set to 5000 bytes and inter request time of 720 seconds was used. Random waypoint mobility was used in this simulation as the model for this work. Random mobility used shows more behavior, good mobility and it was simple to used [16]. The three important configuration parameters used for Wireless LAN, FTP application and Mobility shown in table 2 . 
Table 2: Simulation Configuration And Setup

\begin{tabular}{l|l|}
\hline \multicolumn{1}{c|}{ Attributes } & \multicolumn{1}{c}{ Value } \\
\hline Wireless LAN Parameters \\
\hline $\begin{array}{l}\text { Physical } \\
\text { Characteristics }\end{array}$ & Extended Rate \\
PHY(802.11g) \\
\hline Data Rate & $24 \mathrm{Mbps}$ \\
\hline Transmit Power (watt) & 0.005 \\
\hline Buffer Size (bits) & 256000 \\
\hline $\begin{array}{l}\text { Large Packet } \\
\text { Processing }\end{array}$ & Drop \\
\hline FTP application parameters \\
\hline Inter-Request Time (sec) & Exponential (720) \\
\hline File Size (bytes) & Constant (5000) \\
\hline Type Of Service & Best Effort (0) \\
\hline Random Waypoint Parameters \\
\hline Speed (meters/seconds) & Constant (5) \\
\hline Pause Time (seconds) & Constant (0) \\
\hline Start Time (seconds) & Constant (0) \\
\hline
\end{tabular}

\subsection{Performance Metric}

For comparison between the protocols under the applications generating medium traffic in this paper, a four different metrics have been chosen:

\subsubsection{Data dropped (bits/sec)}

Represent the total size of data packets dropped in the network due to the size of the higher layer packet, which is greater than the maximum allowed data size

\subsubsection{End To End Delay ( $\mathrm{sec}$ )}

Is defined as the average time delay for data packets to reach from the source node to the destination node in the network. It includes processing, queuing and propagation delay of the link [17]. End to end delay can be mathematically be defined by equation 1 :

$$
\begin{aligned}
& \text { Delay } \\
& =\frac{1}{N} \sum_{n=1}^{N}\left(T R_{n}-T S_{n}\right)
\end{aligned}
$$

Where $\mathrm{N}$ represent the total number of data packet received, $\mathrm{TS}_{\mathrm{n}}$ represent the time of $\mathrm{n}$ data packet sent and $\mathrm{TR}_{\mathrm{n}}$ represent the time of $\mathrm{n}$ data packet received.

\subsubsection{Routing Overhead (bits/sec)}

This statistics represents the Total Routing Traffic Sent by all nodes in the network. (Routing traffic send includes: HELLO Messages sent + TC Messages sent + TC Messages forwarded). The routing overhead is estimated as the ratio of the routing packets over the total packets sent.

\subsubsection{Throughput (bits/sec)}

It is the total number of packets successfully transferred from source to destination through the network in a unit period of time (second) [18]. Mathematically, Throughput can be calculated from the following equation 2 :

Throughput

$=\frac{\text { No. of Packets } X \text { packet size } X 8}{\text { time duration }}$

\section{RESULT ANALYSIS}

The main objective of this research paper is an analysis and performance evaluation of the routing protocols AODV, DSR and OLSR by varying the number of nodes in FTP application. The results of this research have been divided on two scenarios, one of them consists of 25 and 35 mobile nodes and the other consists of 85 and 100 mobile nodes. This section examines the effect of number of nodes on network performance of AODV, DSR and OLSR routing protocols, and taken the result in terms of four performance metrics, namely Data Dropped, end to end Delay, Routing overhead and Throughput.

\subsection{The Effect of Nodes' Number on Data Dropped}

Every figure is divided onto two graphs that represent the scenario with different nodes. The graphs of results are presented in a two dimensional Cartesian plane where the horizontal-axis represents the simulation time progress in seconds and the vertical-axis represents performance metric data dropped measured in bits per seconds. Figure 1 shows that the effect of a few density of nodes on delay for 25 and 35 nodes respectively for the first scenario.

Under 25 and 35 nodes in the first scenario, it's obvious that, the increasing in the number of nodes, followed by increasing in the data dropped in all protocols. Figure 1 shows that the data dropped under 25 and 35 nodes is minimum in OLSR protocol as compared to AODV and DSR, whereas the AODV and DSR protocols have the same data dropped under 25 nodes, but under 35 nodes DSR is less than AODV. This concludes that the OLSR protocol performs better than AODV and DSR Protocols.

Figure 2 shows that the effect of a high density nodes on delay under 85 and 100 nodes respectively for the second scenario. The AODV protocol has a maximum data dropped during the simulation time and in the same time DSR has a data dropped, but less than AODV, whereas the OLSR protocol has the lowest data dropped. Finally, the OLSR protocol is the best performance in terms of data dropped with different number of nodes in both scenarios due to its proactive characteristics. 

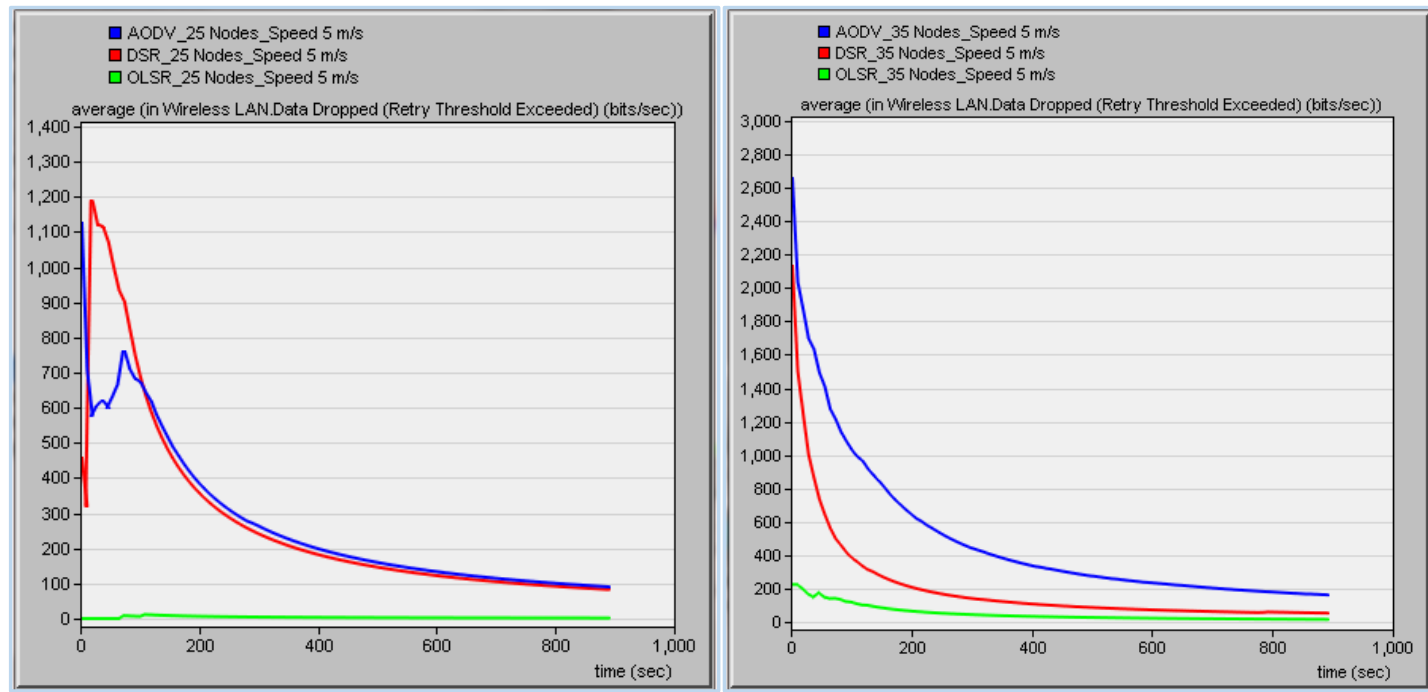

Figure 1: Data dropped under 25 and 35 Nodes versus simulation time

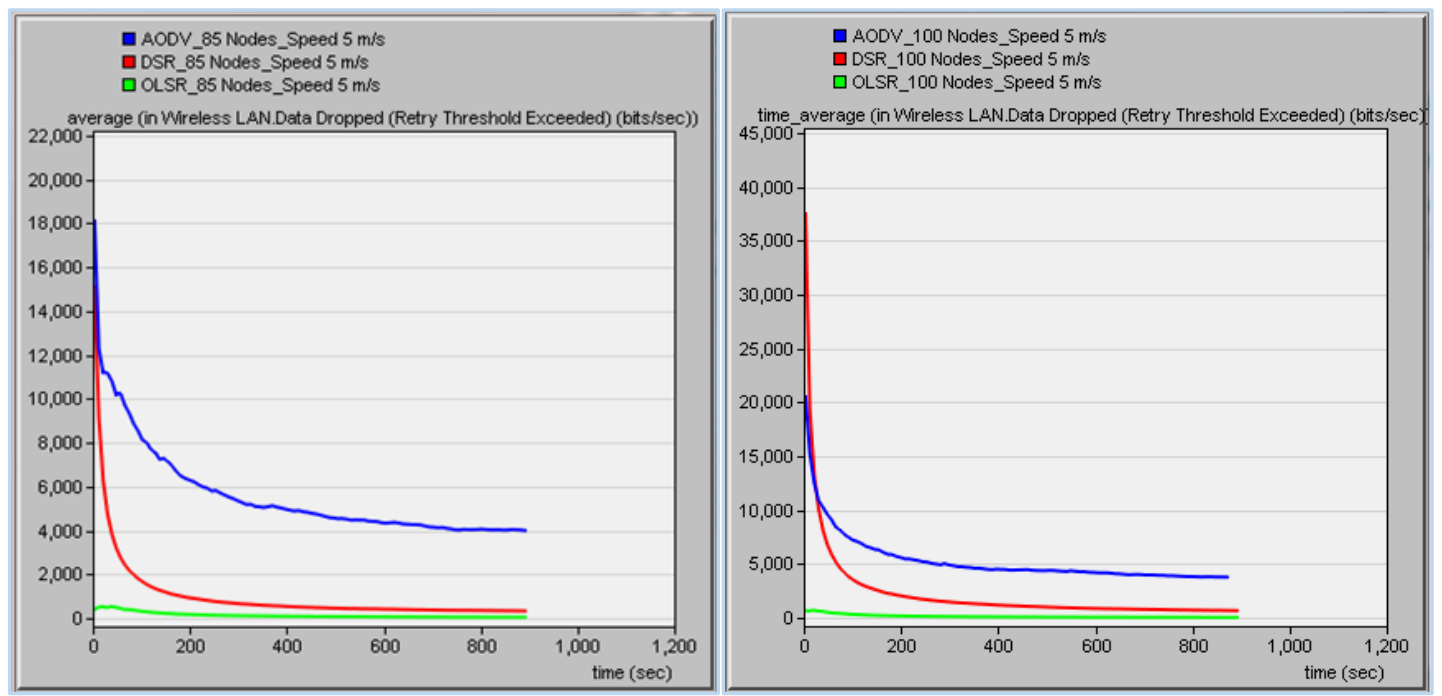

Figure 2: Data dropped under 85 and 100 Nodes versus simulation time

\subsection{The Effect of Nodes' Number on Delay}

This section illustrates the analyzing of the performance for terms wireless LAN delay as shown in figures 3 and 4 for a low density of nodes and a high density of nodes respectively. In both scenarios, the $y$-axis represents the measurement of delay in seconds and also the $\mathrm{x}$-axis represents the simulation time progress in seconds. Figure 3 shows that the AODV protocol has the highest delay and DSR protocol has almost the same performance of AODV in terms of delay under 25 and 35 nodes, but AODV is larger than DSR in terms of average value that shown the result summary in table3. Hence, OLSR protocol has the highest performance and the delay is very low compared to other protocols. The results show that the OLSR protocol has a minimum delay as compared to DSR and AODV protocols under 85 and 100 nodes as shown in figure 4 . According to figures 3 and 4 at low and high density network, the AODV protocol performs worst in terms of end to end delay, due to its reactive nature, in addition to the fixed pause timings parameter.

From this simulation, it's obvious that delay on the OLSR protocol is constant for both scenarios which is very important for communication, whereas delay of DSR and AODV is nonlinear with simulation time. Hence OLSR protocol has the best performance in terms of end to end delay in all scenarios, and this is due to its proactive characteristics. 

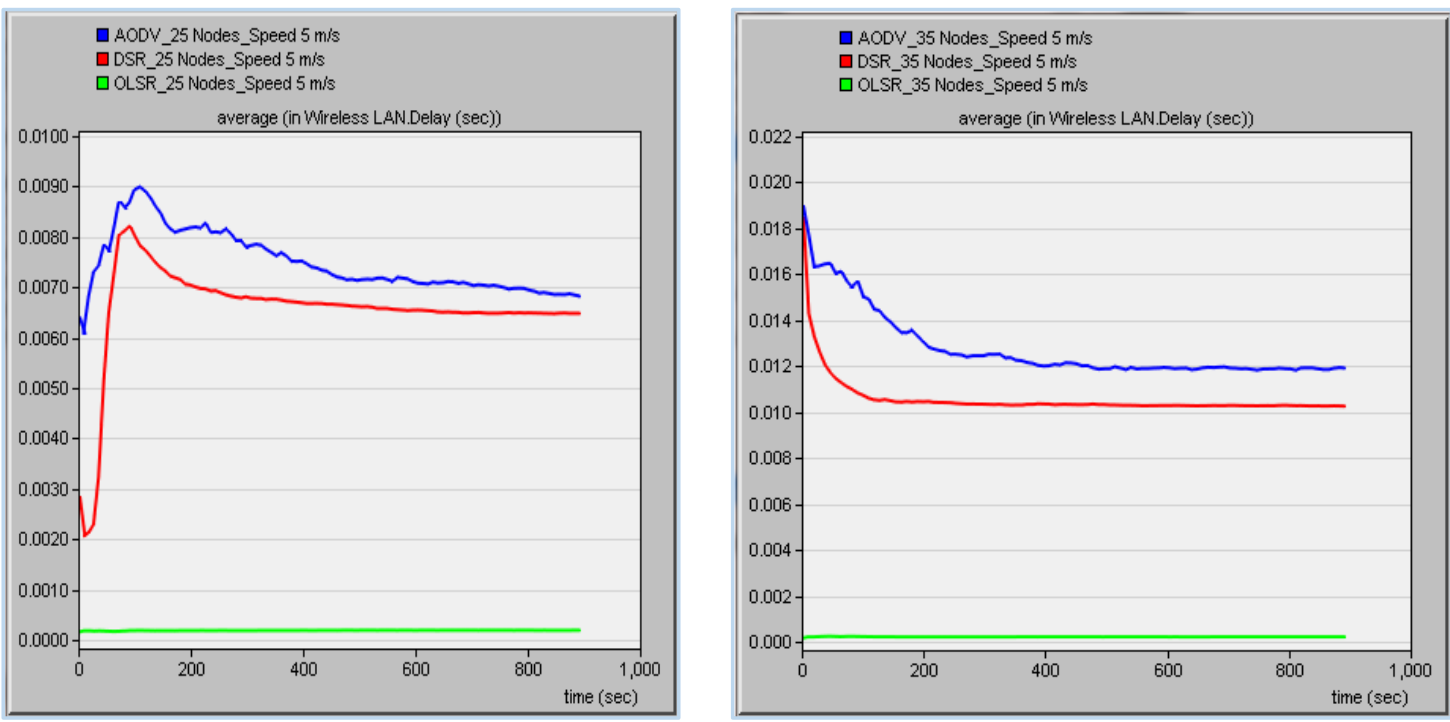

Figure 3: wireless LAN Delay under 25 and 35 Nodes versus simulation time
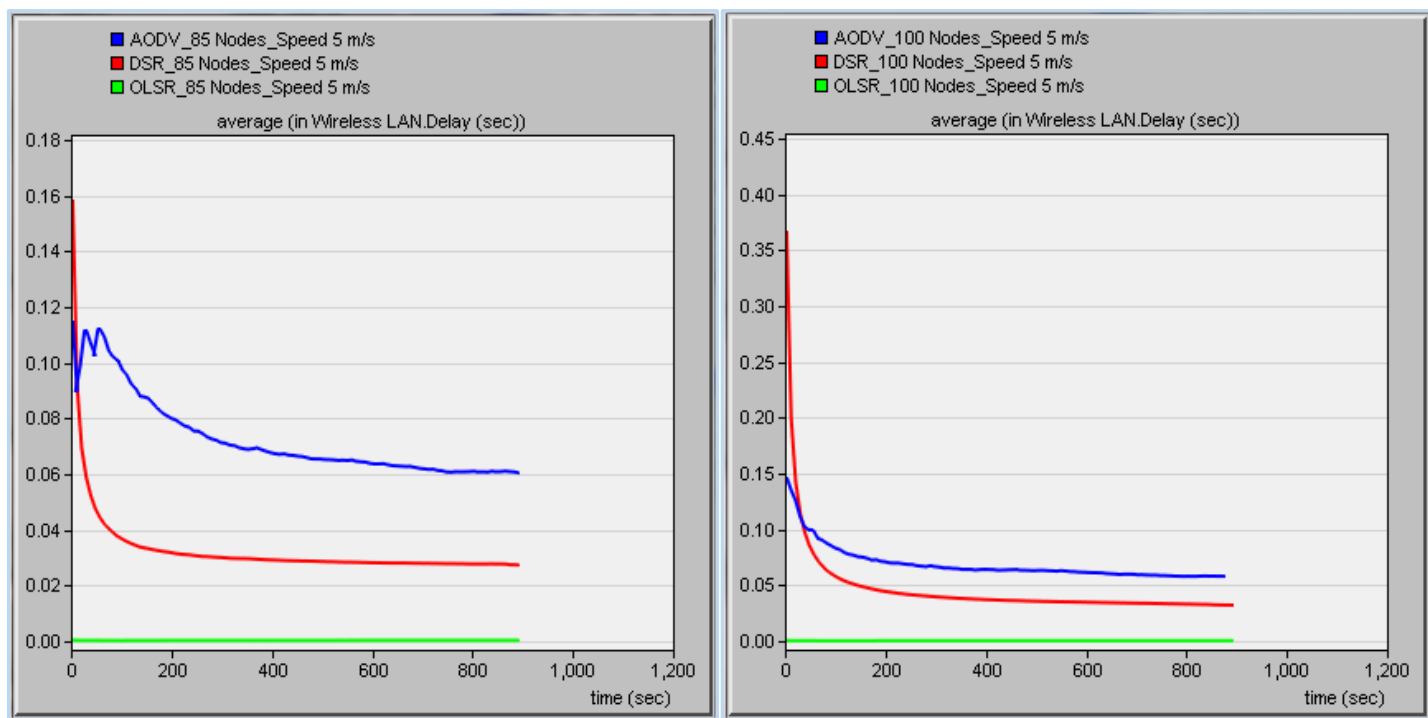

Figure 4: wireless LAN Delay under 85 and 100 Nodes versus simulation time

\subsection{The Effect of Nodes' Number on Routing Overheads}

Routing overhead which is defined as the total number of routing packets transmitted over the network, is an important measure of the scalability of the protocol, and thus the network.

Figures 5 and 6 , present the effect of the number of nodes under two scenarios in routing overhead in bits that required to deliver a data packet. In figure 5 under node density 25 and 35 nodes, it is observed that the routing overhead is a maximum in AODV and a medium in OLSR whereas DSR has a minimum routing overhead. In figure 6 under node density 85 and 100 nodes, AODV protocol has a highest routing overhead as compared to other protocols. DSR protocol has a lowest routing overhead whereas OLSR protocol larger than DSR.

According to figures 5 and 6 , the AODV protocol has the highest routing overhead through the proposed model in all scenarios due to their reactive characteristics, uses multicast category and hello message. In both scenarios, all simulations shown that the routing protocol AODV sends the largest amount of routing traffic in the network followed by OLSR whereas DSR sends the least amount of routing traffic sent. Therefore, the DSR protocol performs better than the AODV and OLSR protocols in terms of routing overhead. 

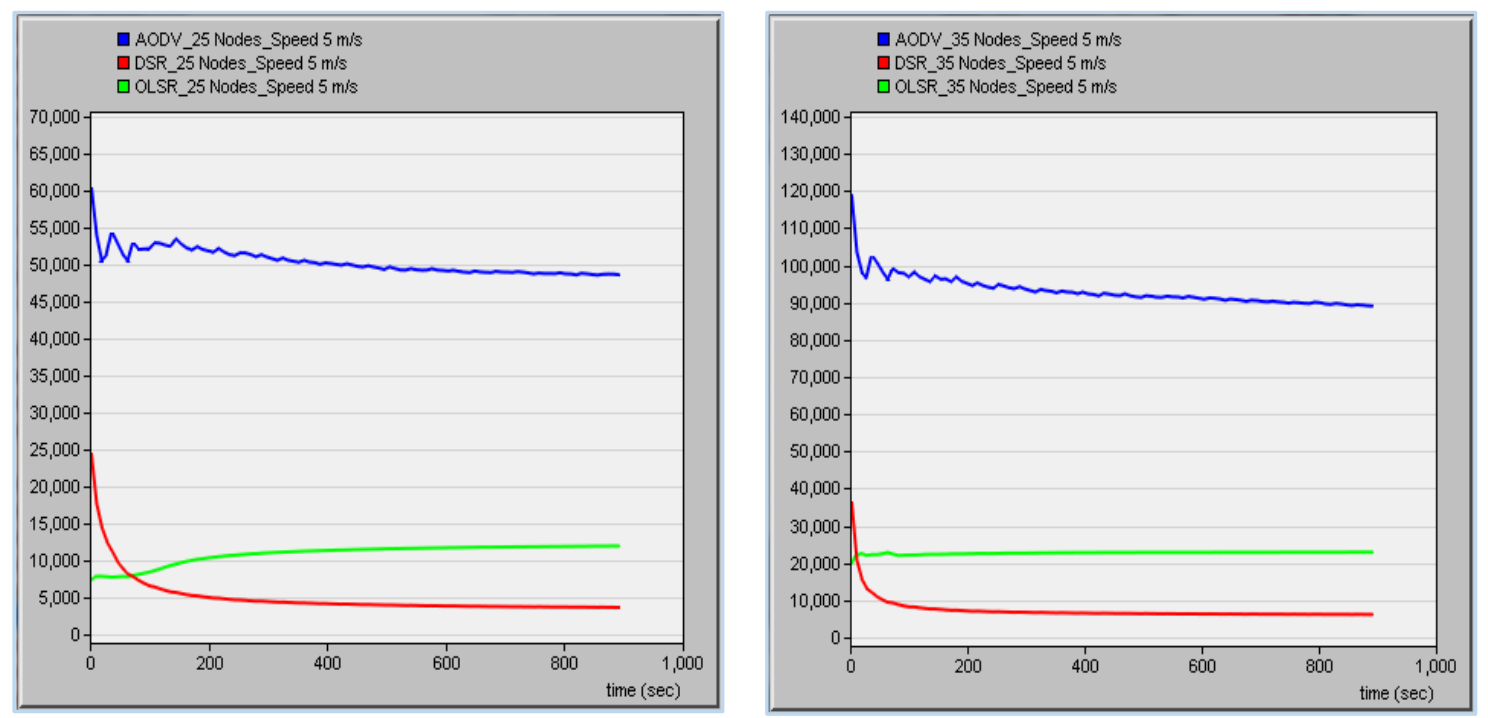

Figure 5: Routing overhead under 25 and 35 Nodes versus simulation time
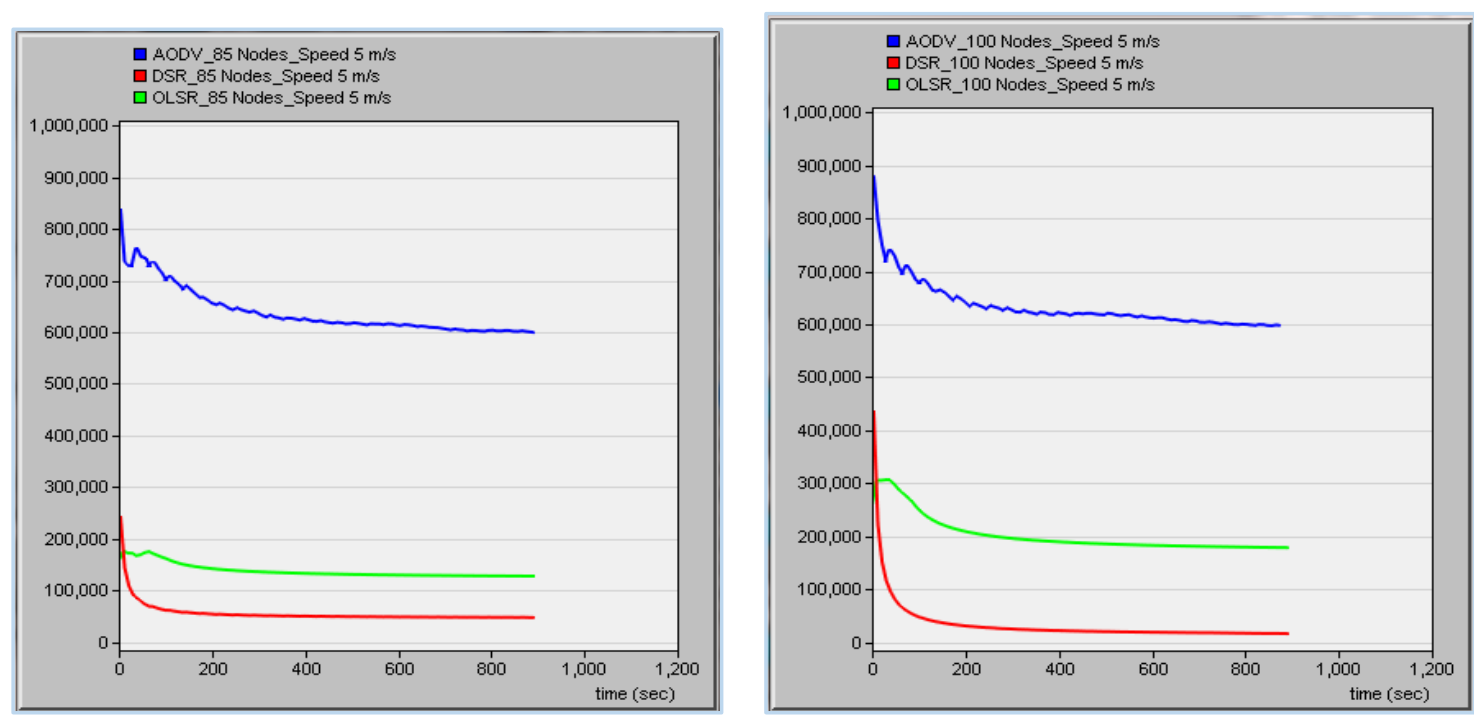

Figure 6: Routing overhead under 85 and 100 Nodes versus simulation time

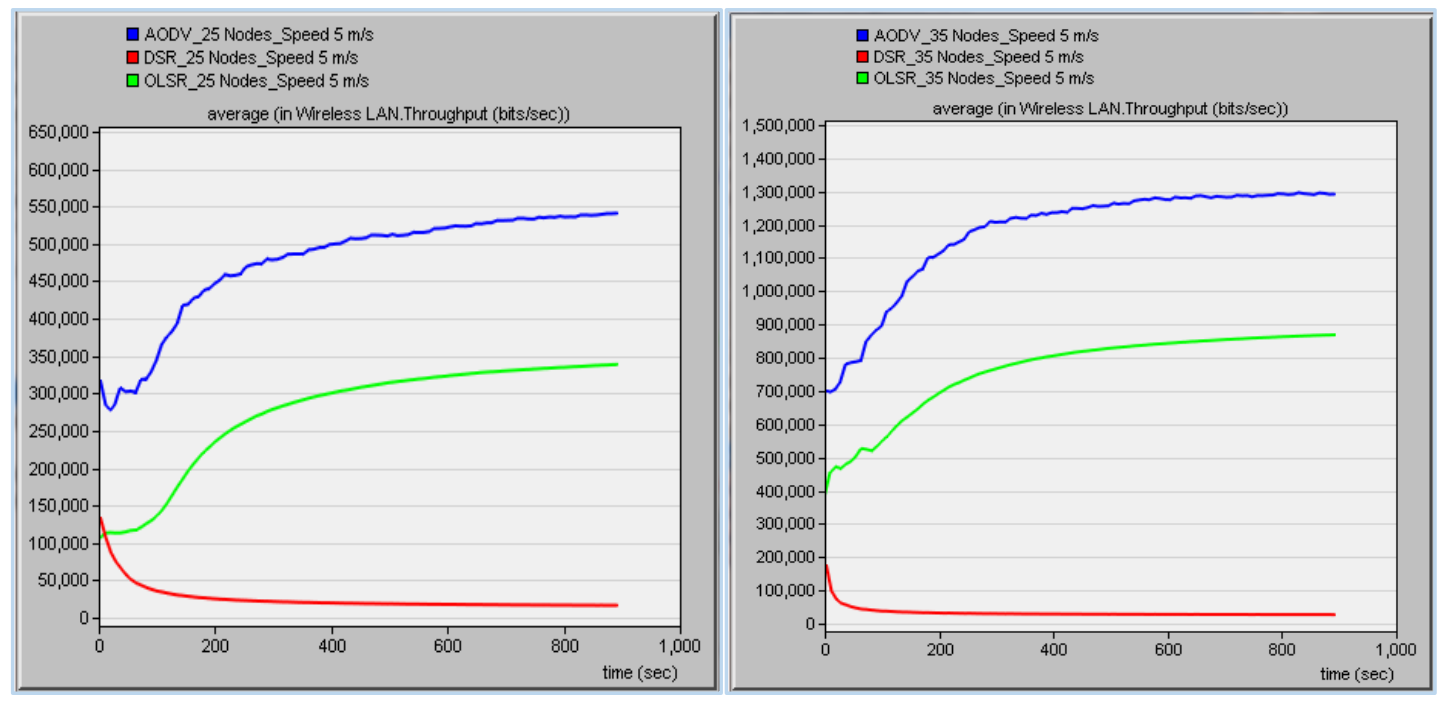

Figure 7: Throughput 25 and 35 Nodes versus simulation time 

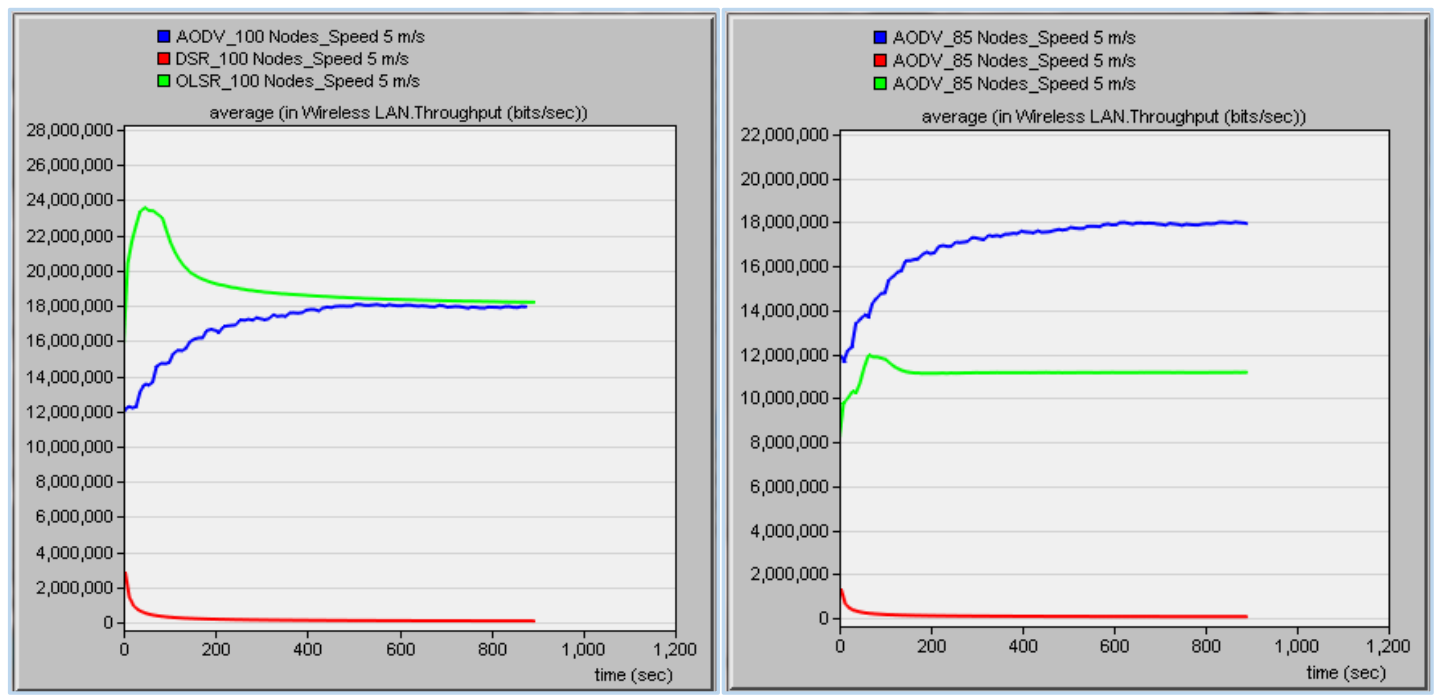

Figure 8: Throughput 85 and 100 Nodes versus simulation time

\subsection{The Effect of Nodes' Number on Throughput}

In this section, we analyze the performance comparison of three routing protocols AODV, DSR and OLSR in terms of throughput with varying the number of nodes of $25,35,85$ and 100 nodes under two different scenarios. In low density network scenario under 25 and 35 nodes, the AODV protocol clearly has a higher throughput, whereas DSR has the lowest and OLSR protocol has a medium throughput that shown in figure 7. In a high density network scenario under 85 nodes, the DSR protocol has the worst throughput when it compared with AODV and DSR protocols, also AODV has a higher throughput whereas, OLSR is very closer with AODV protocols as shown in figure 8 , but when increasing the number of nodes to 100 nodes in the same scenario, The OLSR protocol has a better performance in terms of throughput. From our simulation, it's observed that the performance of AODV protocol is better performance for a low and moderate density networks whereas the OLSR performs well in a high density networks in terms of throughput, also the DSR protocol has the lowest performance in both scenarios.

It can be summarized previous results for the first scenario and the second scenario in this paper in the following tables 3 and 4 respectively after Export all previous data graph from OPNET to spreadsheet.

Table 3: Experiment Result Summary for First Scenario

\begin{tabular}{|c|c|c|c|c|c|}
\hline Nodes No. & Metrics & $\begin{array}{c}\text { Data Dropped } \\
\text { (bits/sec) }\end{array}$ & Delay (sec) & $\begin{array}{c}\text { Routing Overhead } \\
\text { (bits/sec) }\end{array}$ & $\begin{array}{c}\text { Throughput } \\
\text { (bits/sec) }\end{array}$ \\
\hline \multirow{3}{*}{$\mathbf{2 5}$} & AODV & 270 & 0.0075 & 50,358 & 475,820 \\
\cline { 2 - 6 } & DSR & 276 & 0.0065 & 5,058 & 25,493 \\
\cline { 2 - 6 } & OLSR & 3 & 0.0002 & 10,866 & 274,722 \\
\hline \multirow{3}{*}{35} & AODV & 501 & 0.01278 & 93,180 & $1,173,894$ \\
\cline { 2 - 6 } & DSR & 206 & 0.01058 & 7,410 & 33,313 \\
\cline { 2 - 6 } & OLSR & 50 & 0.00021 & 22,660 & 761,770 \\
\hline
\end{tabular}

Table 4: Experiment Result Summary for Second Scenario

\begin{tabular}{|c|c|c|c|c|c|}
\hline Nodes No. & Metrics & $\begin{array}{c}\text { Data Dropped } \\
\text { (bits/sec) }\end{array}$ & Delay (sec) & $\begin{array}{c}\text { Routing Overhead } \\
\text { (bits/sec) }\end{array}$ & $\begin{array}{c}\text { Throughput } \\
\text { (bits/sec) }\end{array}$ \\
\hline \multirow{3}{*}{85} & AODV & 5,640 & 0.07277 & 639,516 & $16,987,872$ \\
\cline { 2 - 6 } & DSR & 1,056 & 0.03303 & 56,752 & 114,624 \\
\cline { 2 - 6 } & OLSR & 133 & 0.00031 & 138,565 & $11,131,291$ \\
\hline \multirow{3}{*}{100} & AODV & 5,335 & 0.06912 & 621,418 & $16,683,765$ \\
\cline { 2 - 6 } & DSR & 2,314 & 0.04753 & 35,362 & 200,709 \\
\cline { 2 - 6 } & OLSR & 142 & 0.00037 & 202,853 & $19,059,836$ \\
\hline
\end{tabular}

\section{CONCLUSION}

This paper examines the impact of nodes number on the performance of three main routing protocols (AODV, DSR and OLSR) for MANETs, under FTP traffic in terms of performance parameters, namely data dropped, end-to-end delay, routing overhead, and throughput using OPNET 
simulator version 17.5. The obtained results show that the performance of each routing protocol depends on all of these parameters. In particular, in terms of Throughput the AODV protocol shows a satisfactory performance, however, for a denser network, the OLSR protocol has a performance better. The OLSR protocol outperforms AODV and DSR protocols by having the lowest value of data dropped and delay in a both proposed scenarios, whereas the DSR protocol outperforms AODV and OLSR protocols in terms of routing overhead by having the lowest bits that require do deliver a data packet due to its reactive characteristics. Finally, it can be concluded from the results that the OLSR protocol is mainly more suitable in networks with higher density and where communication between the nodes is quite frequent.

In our future works, a more comprehensive performance analysis between the three routing protocols will be done by varying the network load, speed of nodes and data packet size.

\section{REFERENCES}

[1] Frey, Michael, Friedrich Grose, and Mesut Gunes. Energyaware ant routing in wireless multi-hop networks. ICC, international conference on IEEE, august 2014. Pages 190-196.

[1] S.Corson and J.Macker. Mobile ad hoc networking (MANET): Routing Protocol Performance Issues and Evaluation Considerations. Available at http://www.ietf.org/rfc/rfc2501.txt.ss

[3] E.M. Royer and C.K. Chai Keong. A Review of Current Routing Protocols for Ad Hoc Mobile Wireless Networks. IEEE Personal Communications Magazine, April 1999, pages 46-55.

[4] Anuradha and Inderjeet Singh Gill. Performance Evaluation of AODV and OLSR for Different Mobility Patterns By Varying Source Nodes in MANET. IJIREEICE, Volume 3, Issue 10, October 2015.

[5] Y.Saadi, S.Kafhali1, A.Haqiq, and B. Nassereddine. Simulation analysis of routing protocols using Manhattan grid mobility mode in MANET. International Journal of Computer Applications. Volume 45, No.23, May 2012, pages 24-30.

[6] G. R .Vijayavani, and G. Prema. Performance comparison of MANET routing protocols with mobility model derived based on realistic mobility pattern of mobile nodes. Advanced Communication Control and Computing Technologies (ICACCCT), 2012 IEEE International Conference on IEEE, 2012.

[7] Tayal, Sandeep, and M. Tripathy. VANET-challenges in selection of vehicular mobility model. Advanced Computing \& Communication Technologies (ACCT), 2012 Second International Conference on. IEEE, 2012.

[8] P. Kuppusamy, K. Thirunavukkarsu, and B. Kalavathi. A study and comparison of OLSR, AODV and TORA routing protocols in ad hoc networks. Presented at 3rd IEEE Conference on Electronics Computer Technology (ICECT 2011), 8-10 April, 2011.

[9] R. Kaur and S. Malhotra. Performance Analysis of Proactive and Reactive Protocols in Mobile Ad hoc Network. International Journal of Advanced Research in Computer Science and Software Engineering, Volume 3, Issue 8, August 2013, pages1424-1430.

[10] H. kaur and E. singh. Performance comparison of OLSR, GRP and TORA using OPNET. International Journal of Advanced Research in Computer Science and Software Engineering, Volume 2, Issue 10, October 2012, pages 260-267.

[11] N. P. Bobade1, and N. N. Mhala. Performance Evaluation of AODV and DSR On-Demand Routing Protocols with Varying MANET SIZE. International Journal of Wireless \& Mobile Networks (IJWMN), Volume 4, No. 1, February, 2012, pages 183-196.

[12] A. Al-Baseera, G.Taliba, and A. Bawazira. Multi-hop Wireless Network: A Comparative Study for Routing Protocols Using OMNET++ Simulator. Journal of Ubiquitous Systems \& Pervasive Networks, Volume 7, No. 1, 2016.

[13] S. Mohapatraa, and P.Kanungob. Performance analysis of AODV, DSR, OLSR and DSDV Routing Protocols using NS-2 Simulator. Elsevier, Volume 30, 2012, Pages 69-76.

[14] P. Mittall, P. Singh and S. Rani. Optimize the routing protocol AODV, OLSR and DSR routing protocols with its performance. International Journal of Advanced Research in Computer and Communication Engineering, Volume 2, issue 9, September 2013, pages 3441-3449.

[15] S. Mittal, P. Kaur. Performance Comparison of AODV, DSR AND ZRP Routing Protocols in MANETs. International Conference on Advances in Computing, Control, and Telecommunication Technologies, in 2009.

[16] A. Aneiba and M. Melad. Performance Evaluation of AODV, DSR, OLSR, and GRP MANET Routing Protocols Using OPNET. International Journal of Future Computer and Communication, Volume 5, No. 1 , February 2016.

[17] M. Gulati and K. Kumar. Performance Comparison of Mobile Ad Hoc Network Routing Protocols. International Journal of Computer Networks \& Communications (IJCNC), Volume 6, No.2, March 2014.

[18] G. Singh, and S. Singh. Evaluation of the effect of Node Movements in Mobile Ad Hoc Networks (MANETs). International Journal of Advanced Research in Computer and Communication Engineering, Vol. 4, Issue 9, September 2015, pages 304-309. 